\title{
The Nonage Concretion-Calcifying Aponeurotic Fibroma
}

\author{
Anubha Bajaj* \\ Histopathologist in A B Diagnostics, New Delhi, India \\ Submission: July 30, 2020; Published: August 28, 2020 \\ *Corresponding author: Anubha Bajaj, Histopathologist in A B Diagnostics, New Delhi, India
}

\section{Preface}

Calcifying aponeurotic fibroma is an exceptional, locally aggressive, benign, fibroblastic, soft tissue neoplasm commonly emerging within palms and soles of young children or adolescents. Calcifying aponeurotic fibroma was denominated as "juvenile aponeurotic fibroma" by Keasbey in 1953 [1]. As the condition incriminates a broad age group, the term "calcifying aponeurotic fibroma" is suggested and preferred. The neoplasm can be contemplated as a cartilaginous analogue of soft tissue fibromatosis. Palisading pattern of fibroblastic dissemination encompass chondroid or calcific foci. The tumour demonstrates a propensity for localized tumour reoccurrence whereas distant metastasis is absent. Calcifying aponeurotic fibroma delineates an aggressive, locally invasive, biological behaviour with an enhanced localized tumour reoccurrence following surgical resection [2]. Clinical and radiographic appearance of calcifying aponeurotic fibroma is variable and contingent to proportionate calcification and soft tissue involvement. The neoplasm is appropriately discerned by cogent histological evaluation [2].

\section{Disease Characteristics}

Calcifying aponeurotic fibroma appears betwixt infancy to 64 years with a median age of disease emergence at 12 years and peak incidence betwixt 8 years to 14 years. Majority of lesions appear within first two decades of life [2]. A male predominance is observed with males being twice as commonly affected as females and a male to female ratio of disease occurrence at 2:1. Calcifying aponeurotic fibroma usually arises adjacent to tendons, fascia, and aponeuroses. Typically, the palms, soles, wrists and ankles are incriminated. The neoplasm can manifest as a dense mass composed of fibrous tissue situated within diverse anatomical locales [2,3]. An estimated 50\% instances demonstrate localized tumour reoccurrence following surgical excision, usually within three years of tumour discernment. Tumour relapse is frequent in children. Localized tumour relapse is enhanced in subjects beneath $<5$ years. Possible tumour reappearance is unrelated to tumour morphology, tumour location or adequacy of primary surgical excision. However, malignant conversion is extremely exceptional $[2,3]$.

\section{Clinical Elucidation}

Calcifying aponeurotic fibroma is a symptom- free neoplasm. The tumefaction manifests as a benign, solitary, miniature, hard, painless, gradually progressive, poorly circumscribed, non tender, soft tissue nodule of several months or years duration, preponderantly arising upon hands or feet. Absence of organ dysfunction or discomfort and minimal functional impairment is delineated. Refractory knee contracture can appear. However, mobility of adjacent joints is unimpaired [3]. Lesions are located upon palm or fingers while involving the hand whereas majority of lesions are situated upon plantar or ankle region, when implicating the foot. Toes are also incriminated [3,4]. Generally, deep-seated, volar fascia, tendons, and aponeuroses of hand or distal upper or lower extremities are incriminated. Calcifying aponeurotic fibroma can also arise from connective tissue of the neck, abdominal wall, lumbar region, knee, thigh, forearm, elbow and arm. Additionally, tumefaction is discerned upon sites such as scalp, gluteal region, popliteal fossa and supraclavicular region $[3,4]$.

\section{Histological Elucidation}

Evaluation of a cogent tissue specimen is crucial for disease discernment. Grossly, multiple, pale to tan, soft tissue fragments or a firm, pale, infiltrative mass of magnitude below $<3$ centimetres is denominated [4]. Macroscopically, poorly defined, firm or rubbery, greyish/ white tumefaction is delineated which is often adherent to circumscribing, dense, fibro- connective tissue such as a tendon, fascia or periosteum. Tumour magnitude ranges from 1.0 centimetre to 5.0 centimetres in maximum diameter. Focal, punctate calcification may be detected, thus engendering a gritty sensation upon the cut surface. Calcifying aponeurotic fibroma demonstrates an ill-defined outline and variable consistency of rubbery to firm $[4,5]$. A nodular or infiltrative, grey/ white, gritty mass is exemplified within the subcutaneous tissue. A tendon may 


\section{Cancer Therapy \& Oncology International Journal}

appear as calcified. The nodule is usually beneath $<3$ centimetres when confined to hands or feet although can be enlarged when appearing in adjunctive sites [4].

On cytological evaluation, benign appearing spindle-shaped cells, chondroid cells, multinucleated giant cells and calcified debris is exemplified [4,5]. The neoplasm characteristically depicts a palisading, peripheral aggregation of spheroidal cells intermixed with fibroblasts. Distinctive foci of calcification are discerned. A benign, fibrous lesion with abundant calcification is denominated which circumscribes foci of fibrous connective tissue composed of spindle- shaped fibroblasts [5]. Classically, the lesion is configured of dual cellular components. Nodular calcific deposition is circumscribed by a palisade of spheroidal, chondrocyte- like cells configuring abridged, parallel arrays. Minimally cellular, spindle-shaped, fibroblastic component is intermixed with coalescent, calcific nodules wherein fibroblastic cellular cords emanate into encompassing soft tissue [4,5].

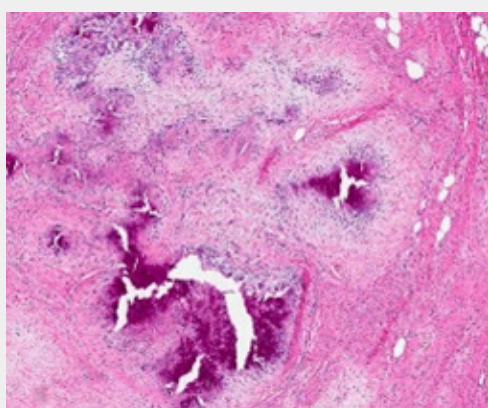

Figure 1: Calcifying aponeurotic fibroma depicting focal calcification, spindle- shaped and epithelioid cells intermixed within a fibrous stroma [9].

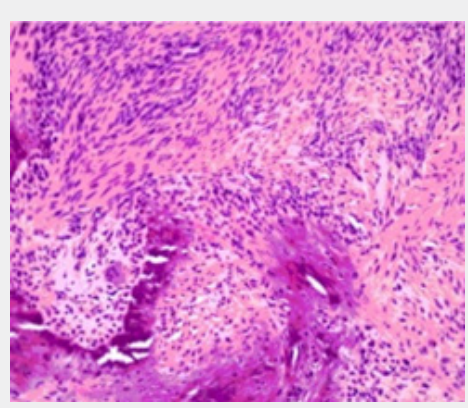

Figure 2: Calcifying aponeurotic fibroma delineating abundant calcification intermingled with a cellular, fibroblastic stroma and an epithelioid cell component with absent mitosis [10].

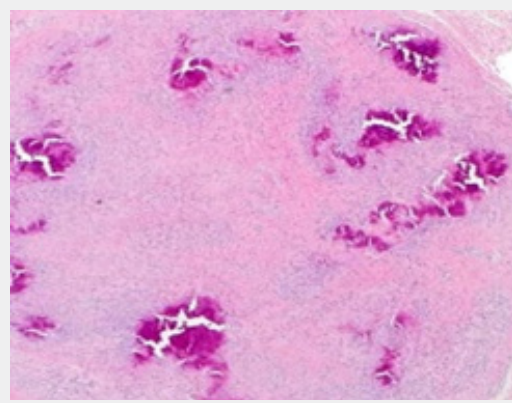

Figure 3: Calcifying aponeurotic fibroma exhibiting focal calcification with prominent, spindle-shaped and epithelioid cell stroma, chondroid foci and lack of atypia [11].

Tumour nodules are enveloped within a hyalinised stroma which may depict chondroid-like features. Spindle-shaped fibroblasts demonstrate a fascicular growth pattern and are intermingled with scattered epithelioid cells. Aforesaid cellular component is arranged at the perimeter of foci of chondroid tissue which may or may not depict mineralization. Plump, spindle- shaped cells with spherical to ovoid nuclei and indistinct cytoplasm are discerned. Cells can simulate chondrocytes. Osteoclast- like giant cells can be frequent or occasional. Intervening stroma is dense and collagenous $[5,6]$. Nodules are composed of plump or epithelioid fibroblasts which palisade around foci of cartilage. Spotty calcification is observed. Tumour cells demonstrate an 


\section{Cancer Therapy \& Oncology International Journal}

indistinct, variable cytoplasm besides plump, elliptical nuclei with vesicular chromatin, condensed beneath the nuclear membrane. Fibrocytes disseminated within the nodular stroma delineate dense, evenly dispersed chromatin [5,6]. Mitotic figures are exceptional and cellular or nuclear atypia is absent. The neoplasm displays enhanced cellularity when emerging within very young subjects. Foci of calcification may be absent in preliminary stage of calcifying aponeurotic fibroma [6].

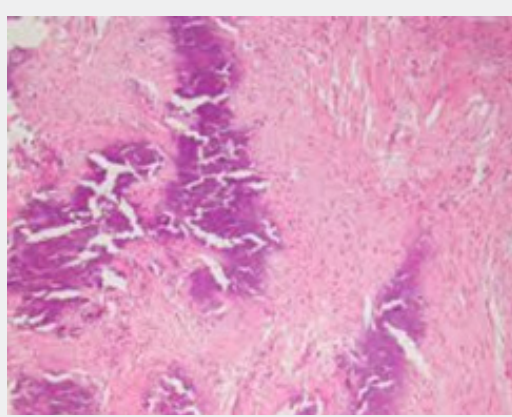

Figure 4: Calcifying aponeurotic fibroma enunciating significant focal calcification and an enveloping stroma composed of mesenchymal, spindle-shaped and epithelioid cells [12].

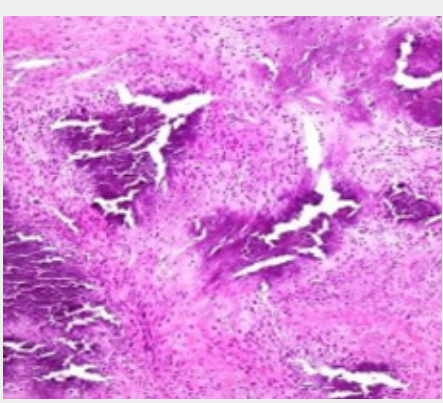

Figure 5: Calcifying aponeurotic fibroma displaying several foci of calcification with an encompassing fibroblastic stroma constituted by fibroblasts and epithelioid cells [13].

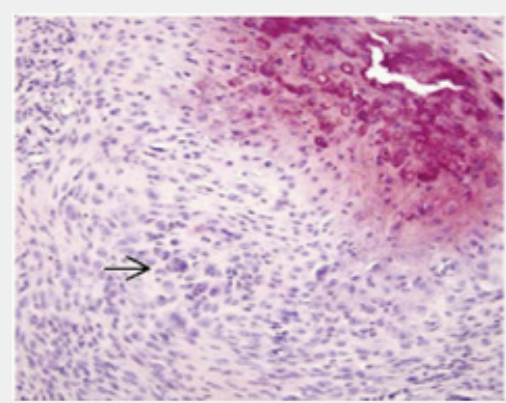

Figure 6: Calcifying aponeurotic fibroma exhibiting focal calcification and an enveloping spindle -shaped cellular component with lack of atypia and mitosis [14].

Tumour nodules can infiltrate peripheral adipose tissue or striated muscle fibres. Aforesaid manifestation is common in infants and young children. Calcification and cartilage are prominent in neoplasms appearing within older children and young adults [6]. Excessive fibrous tissue can be enunciated. As the neoplasm is microscopically infiltrative, circumscribing adipose tissue can be invaded. Osteoclastic cells may appear at the perimeter of calcium deposits. Inflammatory cells are exceptionally delineated [6,7]. Calcifying aponeurotic fibroma demonstrates an initial evolutionary stage of rapid tumour progression and infiltration and a delayed stage with prominent calcification [6]. On ultrastructural analysis, chondrocytes, fibroblasts, and occasional myo-fibroblasts can be observed [7].

\section{Immune Histochemical Elucidation}

Calcifying aponeurotic fibroma is immune reactive to vimentin, CD99, CD68 and S100 protein. Calcifying aponeurotic fibroma is focally immune reactive to muscle specific actin (MSA+/-) and smooth muscle actin (SMA+/-). The fibroblasts are immune reactive to smooth muscle actin (SMA) and immune non- 


\section{Cancer Therapy \& Oncology International Journal}

reactive to beta catenin $[3,4]$. Calcifying aponeurotic fibroma is immune reactive to muscle specific actin (MSA) in an estimated $50 \%$ instances and smooth muscle actin (SMA) in around 50\% subjects. The neoplasm is usually immune non-reactive to CD34, CD57 or progesterone receptor (PR) [3,4]. On cytogenetic analysis, the benign tumour can enunciate aneuploidy [3].

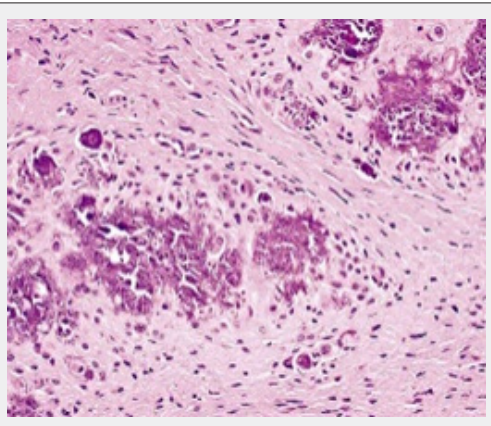

Figure 7: Calcifying aponeurotic fibroma depicting random foci of calcification admixed with spindle-shaped, fibroblastic and epithelioid cell component with a myxoid stroma [15].

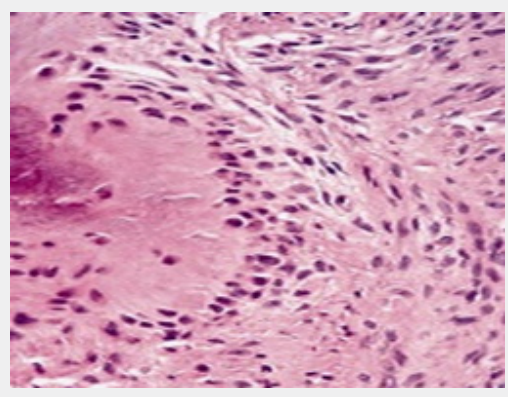

Figure 8: Calcifying aponeurotic fibroma delineating focal calcification with palisading spindle and epithelioid cells and a circumscribing mesenchymal, fibromatous stroma [15].

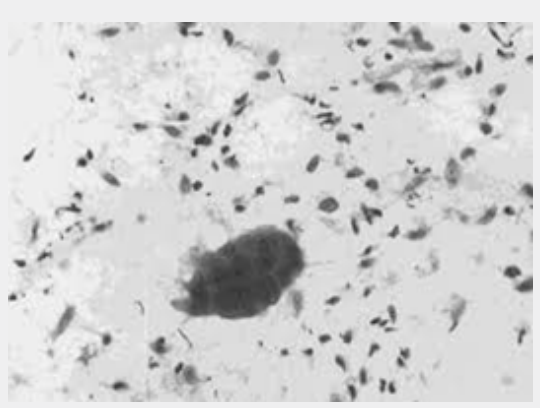

Figure 9: Cytological appearance of calcifying aponeurotic fibroma depicting focal calcification and an intermixed spindle cells and fibrocytes dispersed within a myxoid stroma [16].

\section{Differential Diagnosis}

Calcifying aponeurotic fibroma requires a demarcation from benign conditions such as rheumatoid nodule, chondroma of soft parts, fibrous hamartoma of infancy, Schwannoma, and fibromatosis [2,3]. Chondroma of soft parts can incriminate the hands. The tumour is well circumscribed and demonstrates extensive chondroid differentiation. Tumour infiltration of adjacent soft tissue or circumscribing epithelioid cell component is absent [3,4]. Fibrous hamartoma of infancy exhibits immature mesenchyme and fibroblasts configuring a trabecular architecture. However, cellular palisading, cartilage fragments or focal calcification is absent. The lesion spares hands or feet $[3,4]$. Infantile fibromatosis is usually discerned upon head, neck or proximal extremities and appears in infants. Hands or feet of children or young adults do not exhibit the lesion. Intermingling stroma is myxoid, rather than chondroid. Calcification is exceptional $[3,4]$. Superficial palmar or plantar and desmoid fibromatosis are devoid of foci of calcification or chondroid differentiation [3]. Histological segregation is required from fibroma or dermatofibroma, predominantly due to abundant fibrous tissue component. Also, distinction from soft tissue neoplasms such as synovial sarcoma is mandated and challenging $[3,4]$. 


\section{Cancer Therapy \& Oncology International Journal}

\section{Investigative Assay}

On plain radiography, calcifying aponeurotic fibroma exhibits a soft tissue mass with foci of fine or coarse calcification. Bony incrimination appears as periosteal reaction or defect in the cortical bone $[7,8]$. Computerized tomography (CT) is advantageous in visualizing tumour associated calcification [7]. Magnetic resonance imaging (MRI) is an accurate modality which can be employed in the evaluation of soft tissue neoplasms. The technique is especially adopted for preoperative planning besides assessment of expanse and perimeter of the tumefaction. Signal intensity of the tumefaction is contingent to quantity of calcification and fibrous tissue component [7,8]. Calcifying aponeurotic fibroma represents a heterogeneous mixture of enhanced and minimal signal intensity upon T2 weighted imaging and minimal to intermediate signal intensity upon T1 weighted imaging with intense contrast enhancement [7,8]. Magnetic resonance imaging (MRI) demonstrates a mass with minimal signal intensity, in contrast to muscle, with T1 weighted imaging, moderately enhanced signal intensity upon T2 weighted imaging and an intense, heterogeneous enhancement with gadolinium contrast upon T1 weighted fat- suppressed imaging [7,8]. Typical imaging features of fibrous tissue with minimal T1 and T2 signal intensities and mild enhancement within post contrast imaging can be exemplified. However, aforesaid, manifestations are nonspecific for the discernment of calcifying aponeurotic fibroma $[7,8]$.

\section{Therapeutic Options}

As a predilection for localized tumour reoccurrence is observed, surgical excision with a wide, tumour-free perimeter is necessitated. Conservative surgical extermination during the delayed phase is a preferred therapy to maintain adequate organ function $[7,8]$. Comprehensive surgical eradication of the tumefaction is a preferred therapeutic modality. Close monitoring of surgically excised neoplasm is mandated as localized tumour relapse occurs in > half the neoplasms. Conservative therapy with adequate surgical excision is appropriate. Re-excision can be necessarily employed for treating tumour reoccurrence $[7,8]$.

\section{References}

1. Keasbey LE (1953) Juvenile aponeurotic fibroma (calcifying fibroma)a distinctive tumour arising in the palms and soles of young children. Cancer 6(2): 338-346.

2. Allen PW, Enzinger FM (1970) Juvenile aponeurotic fibroma. Cancer 26: 857-867.

3. Garcia Navas FM, Fernandez N, Alberto Lopez, Pedro Montero (2019) Calcifying aponeurotic fibroma of the sole of the foot in an elderly patient. Foot 40: 64-67.

4. Zhang L, Kobayashi H, Masachika Ikegami, Takahiro Ohki, Yusuke Shinoda, et al. (2020) Calcifying aponeurotic fibroma of the thigha differential diagnosis for infant knee contracture and leg length inequality (a case report). Int J Surg Case Rep 69: 96-100.

5. Hamoud AM, Nasser AY, Alshamlan Najd Abdulrahman (2020) Calcifying aponeurotic fibroma around Achilles tendon - a case report. Radiol Case Rep 15(6): 753-756.

6. Motta F, Scavo S, G M Vecchio, G Fuccio-Sanzà, F Nicolosi, et al. (2018) Calcifying aponeurotic fibroma - a core biopsy-based diagnosis. Pathologica 110(4): 307-312.

7. Takaku M, Hashimoto I Hideki Nakanishi, Taeko Kurashiki (2011) Calcifying aponeurotic fibroma of the elbow- a case report. J Med Invest 58(1-2): 159-162.

8. Cho YH, Ahn KS, Chang Ho Kang, Chul Hwan Kim (2015) Calcifying aponeurotic fibroma of the dorsum of the foot - radiographic and magnetic resonance imaging findings in a four-year-old boy. Iran J Radiol 12(2): e23911.

9. Image 1 Courtesy: Paediatric Orthopaedic Pathology

10. Image 2 Courtesy: Science direct

11. Image 3 Courtesy: Twitter

12. Image 4 Courtesy: Dermnet NZ

13. Image 5 Courtesy: Webpathology

14. Image 6 Courtesy: Basic Medical Key

15. Image 7 and 8 Courtesy: Pathology Outlines

16. Image 9 Courtesy: Wiley online library

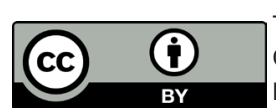

This work is licensed under Creative Commons Attribution 4.0 License DOI:10.19080/CTOIJ.2020.16.555948
Your next submission with Juniper Publishers will reach you the below assets

- Quality Editorial service

- Swift Peer Review

- Reprints availability

- E-prints Service

- Manuscript Podcast for convenient understanding

- Global attainment for your research

- Manuscript accessibility in different formats

( Pdf, E-pub, Full Text, Audio)

- Unceasing customer service

Track the below URL for one-step submission https://juniperpublishers.com/online-submission.php 